Supporting Information for:

\title{
Time-Resolved Broadband Cavity-Enhanced Absorption Spectroscopy behind Shock Waves
}

Akira Matsugi, ${ }^{* \dagger}$ Hiroumi Shiina, ${ }^{\dagger}$ Tatsuo Oguchi ${ }^{\ddagger}$, and Kazuo Takahashi ${ }^{\S}$

${ }^{\dagger}$ National Institute of Advanced Industrial Science and Technology (AIST), 16-1 Onogawa, Tsukuba, Ibaraki 305-8569, Japan.

$\$$ Department of Environmental and Life Sciences, Toyohashi University of Technology, 1-1 Hibarigaoka, Tenpaku-cho, Toyohashi 441-8580, Japan.

${ }^{\S}$ Department of Materials and Life Sciences, Sophia University, 7-1 Kioi-cho, Chiyoda-ku, Tokyo 102-8554, Japan. 

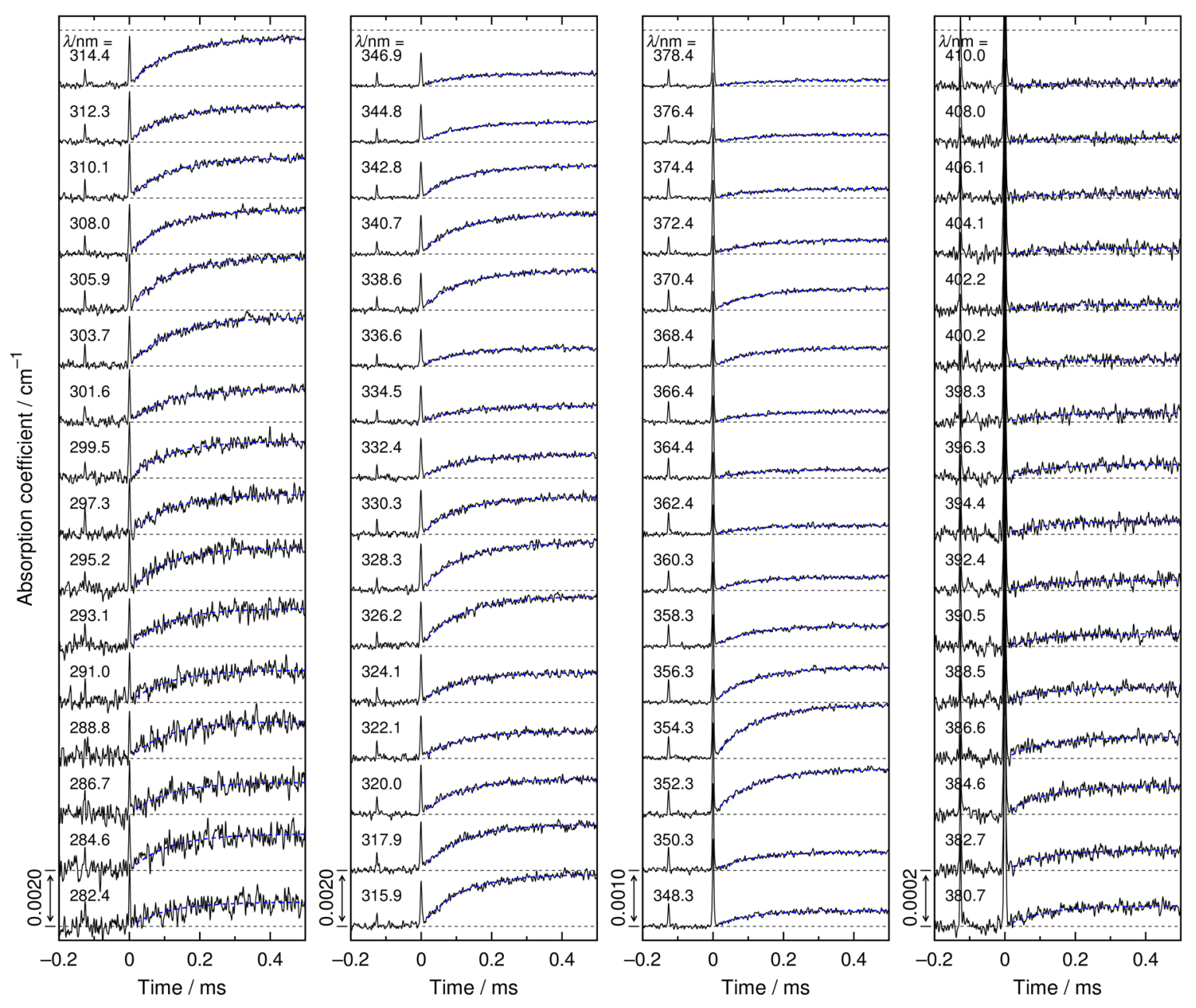

Figure S1. Wavelength-specific time profiles of the absorption coefficients observed following the pyrolysis of 1,3,5-trioxane at $T_{5}=955 \mathrm{~K}$. The dashed lines are the fitted profiles. 

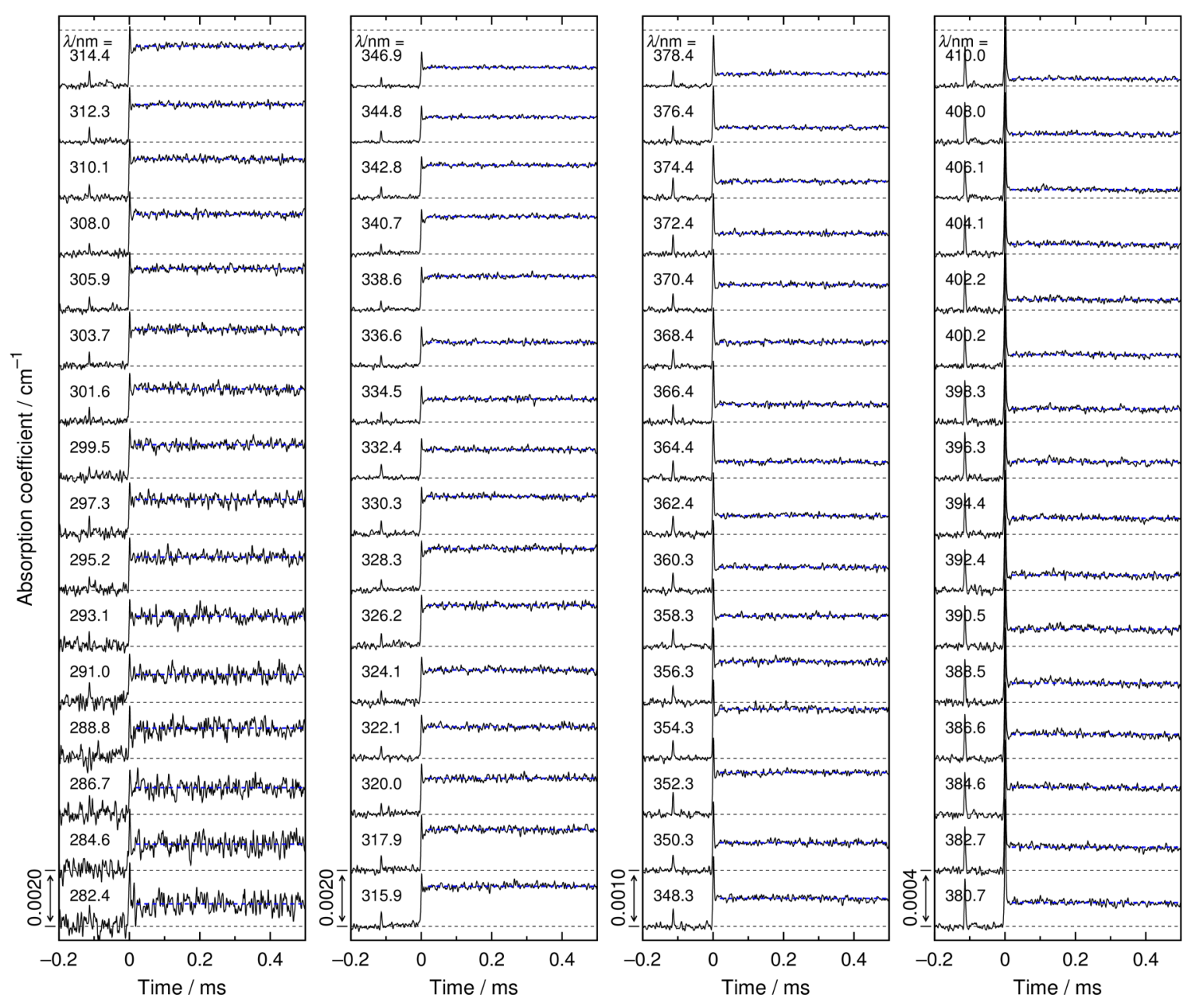

Figure S2. Wavelength-specific time profiles of the absorption coefficients observed following the pyrolysis of 1,3,5-trioxane at $T_{5}=1265 \mathrm{~K}$. The dashed lines are the average of the post-reflected-shock absorption coefficients. 

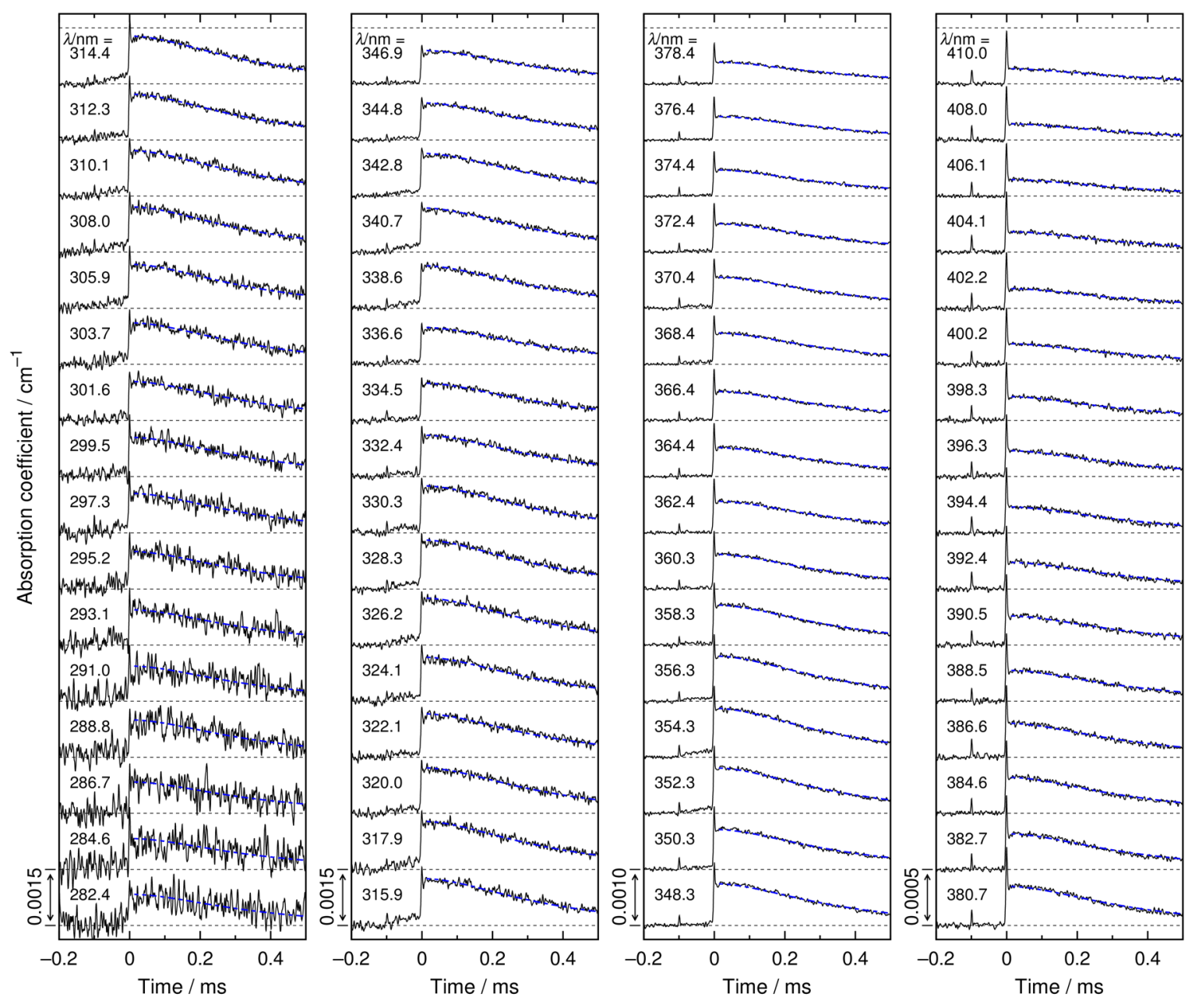

Figure S3. Wavelength-specific time profiles of the absorption coefficients observed following the pyrolysis of 1,3,5-trioxane at $T_{5}=1708 \mathrm{~K}$. The dashed lines are the simulated profiles (see text). 


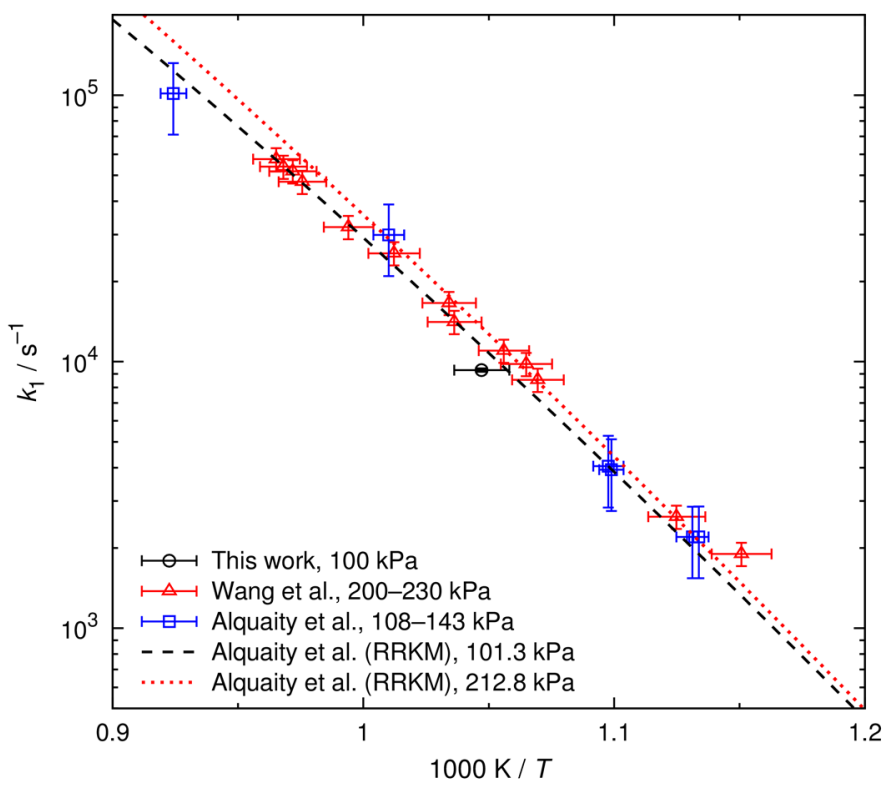

Figure S4. Comparison of the present and literature rate constants for the decomposition of 1,3,5-trioxane (R1).

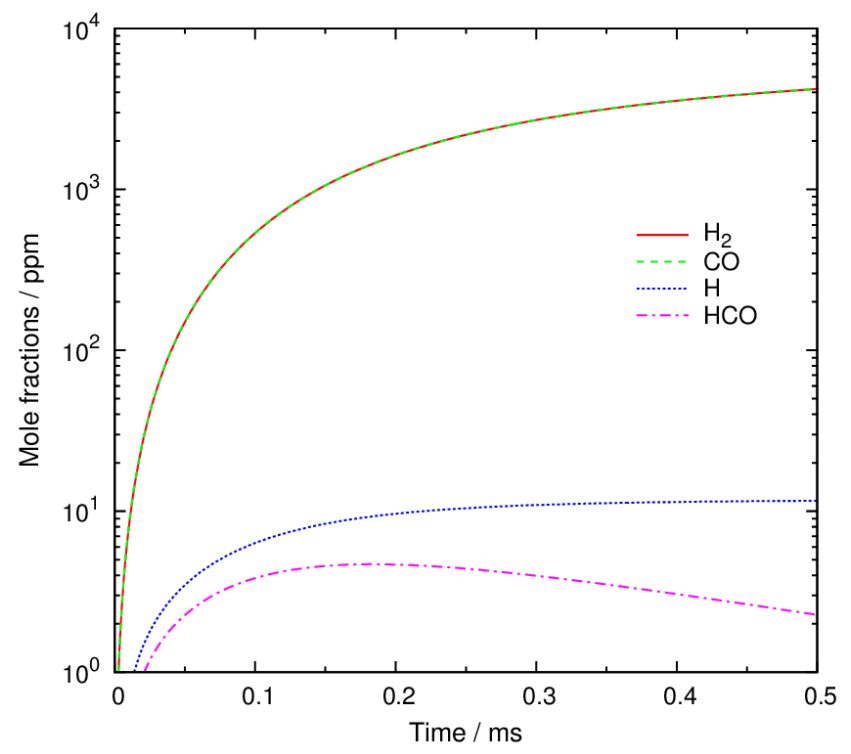

Figure S5. Simulated time profiles of the products from the decomposition of formaldehyde at $1708 \mathrm{~K}$ (note that the $\mathrm{H}_{2}$ and $\mathrm{CO}$ profiles are superimposed). 